\title{
The $\Delta$ Contribution to the Parity-violating Nucleon-nucleon Force
}

\author{
Yan-Rui Liu周 and Shi-Lin Zhu周 \\ ${ }^{1}$ Institute of High Energy Physics, P.O. Box 918-4, Beijing 100049, China \\ ${ }^{2}$ Department of Physics, Peking University, Beijing 100871, China
}

\begin{abstract}
Because the nucleon may be excited and transformed into a virtual $\Delta$ resonance easily, we consider the decuplet contribution to the parity-violating (PV) nucleon-nucleon interaction in the chiral effective field theory. The effective PV nucleon-nucleon potential is derived without introducing any unknown coupling constants.
\end{abstract}

PACS numbers:

\section{INTRODUCTION}

The strangeness-changing weak processes $(\Delta S=1)$ can be studied both in the semi-leptonic decays and strangeness-changing hadronic weak interactions. In contrast, the nuclear parity violation is almost the unique way to study the $\Delta S=0$ hadronic weak interaction experimentally. Up to now, our knowledge on such processes is still relatively poor.

The search for nuclear parity violation [1] started shortly after the observation of the parity violation in nuclear beta decay [2]. Thereafter, there had been many experimental investigations of nuclear parity-violation such as the polarized proton-nucleus scattering, radiative $n p$ capture, $\gamma$ decay of nuclei, neutron spin rotation, and atomic parity-violation [3, 4, 5, 6].

The parity-violating (PV) effect is very small in nuclear processes. Such an effect can be measured through the asymmetry of the cross-sections in the polarized experiments. In some heavy nuclei, there exist two energy levels with different parity which are very close to each other. The PV weak interaction mixes these two levels. Then the asymmetry may be amplified.

However, the few-body nuclear system provides a much cleaner place to study nuclear parity-violation though the asymmetry is only $\sim 10^{-7}$. Experimental progress in this field is very encouraging. Several years ago, the longitudinal analyzing power of $\overrightarrow{p p}$ scattering was measured at TRIUMF [7, 8]. There are also on-going experiments to measure the photon asymmetry in radiative $\vec{n} p$ capture at LANSCE [9], the helicity asymmetry in the photodisintegration of deuterium at IASA [10], and the spin rotation of polarized neutrons in ${ }^{4} \mathrm{He}$ at NIST [11, 12].

Strong interaction dominates the nucleon-nucleon interaction, which is repulsive at the short range. Therefore the weak interactions between nucleons mediated directly by $\mathrm{W}$ and $\mathrm{Z}$ bosons are strongly suppressed since the interaction range is around $0.002 \mathrm{fm}$. On the other hand, the meson nucleon interaction vertex can be parityviolating. Thus one can study nuclear parity violation

\footnotetext{
*Electronic address: yrliu@ihep.ac.cn

${ }^{\dagger}$ Electronic address: zhusl@phy.pku.edu.cn
}

after replacing one strong vertex by the weak one in the meson exchange model.

Historically, the study of nuclear parity-violation with the meson-exchange model started in 1964 [13]. Later, nuclear parity-violation was extensively studied in this framework [14, 15, 16]. In 1980, Desplanques, Donoghue, and Holstein (DDH) investigated the PV nuclear force in a general way and considered the exchanged mesons up to $\rho$ and $\omega[17]$. The DDH method has become a standard way in analyzing experiments since then. In that paper, the PV vertices were parameterized with seven coupling constants: $h_{\pi}^{1}, h_{\rho}^{0,1,2}, h_{\omega}^{0,1}$ and $h_{\rho}^{1 \prime} . h_{\rho}^{1 \prime}$ was found to be small and usually neglected [18]. DDH estimated these coupling constants using the quark model and $S U(6)_{w}$ symmetry. They gave reasonable ranges for the couplings and presented their best guesses. Surprisingly, various experimental constraints are more or less consistent with these DDH estimates, except that the bound on $h_{\pi}^{1}$ from nuclear anapole moment in Cesium [19] does not agree well with those from other experiments [20].

In the past decades, there has been important progress in the study of parity violation both in the single-nucleon case [21, 22, 23, 24, 25] and $N N$ system [26, 27, 28, 29, 30]. In order to investigate nuclear parity violation in a model-independent way, Zhu, Maekawa, Holstein, Ramsey-Musolf and van Kolck reformulated the $\mathrm{PV}$ nucleon-nucleon interaction in the framework of effective field theory (EFT) [31]. At very low energy, the momenta of external fields are very small and the pion can be integrated out. EFT without explicit pions is appropriate. When the external momenta are comparable with the pion mass, EFT with explicit pions is necessary.

For the description of PV NN forces in EFT with explicit pions, the treatment is similar to the study of parity-conserving (PC) NN force in EFT 32, 33]. One simply replaces one $\mathrm{PC}$ vertex with one $\mathrm{PV}$ vertex and imposes chiral symmetry on the PV vertex. In Ref. [31], the $\mathrm{PV}$ potential was calculated to $\mathcal{O}(Q)$ in Weinberg's power counting where $Q$ is the typical scale of the processes. The leading order $\left(\mathcal{O}\left(Q^{-1}\right)\right)$ result reproduced the pion exchange part of DDH formalism. At the next leading order $\left(\mathcal{O}\left(Q^{0}\right)\right)$, explicit computation shows there is in fact no contribution. At the third order, the short range potential was described with contact interactions. The medium range potential was deduced from two-pion 
exchange interactions while the long range potential was obtained by considering corrections to the one-pion exchange interaction. In this framework, Ref. 34] studied a minimal set of parameters to describe low-energy PV observables. In Ref. [35], the authors studied PV asymmetry in $n p \rightarrow d \gamma$ within EFT.

The decuplet baryon $\Delta$ couples to $N \pi$ strongly. The virtual $\Delta$ may aslo contribute to the PV nucleon-nucleon interactions, which was noted long time ago in Refs. [36, 37, 38]. The DDH formalism was extended to investigate the effects due to $\Delta[39]$. With development of the modern EFT language, we will extend the former work [31] and calculate the PV potential by considering $\Delta$ as an explicit degree of freedom in EFT in the present work. The present work was part of Y. R. Liu's thesis submitted in April, 2007. It is interesting to note that an independent work dealing with similar topics appeared recently [40]. However the way to derive the potential in this work is different from that in Ref. [40]. In a recent work [41], the calculation of the longitudinal asymmetry in $p p \rightarrow p p$ by including $2 \pi$ exchange effects which include $N N$ and $N \Delta$ intermediate states is presented.

In order to include the $\Delta$ degree of freedom systematically, we employ the heavy baryon chiral perturbation theory with $\Delta$. The expansion scheme was called the small scale expansion (SSE) [42], which was widely used to study the processes involving $\Delta$ [43, 44, 45, 46, 47, 48, 49, 50, 51]. Both the pion mass and the mass difference between nucleon and $\Delta$ isobar are counted as the order $\mathcal{O}(Q)$. We use this formalism to calculate the $\Delta$ contribution to the $\mathrm{PV}$ NN potential. In the following section, we present the relevant Lagrangian. In Section III, we calculate PV potentials due to the virtual $\Delta$ baryon. The final section is a short summary.

\section{LAGRANGIANS}

In the EFT study of the nucleon-nucleon potential, one performs a systematic expansion of Lagrangians and amplitudes [32, 33]. We present relevant Lagrangians $\mathcal{L}^{(\nu)}$ in this section. They are grouped with chiral in$\operatorname{dex} \nu=d+f / 2-2$ where $d$ is the number of derivatives and powers of the pion mass and $f$ the number of fermion fields. When we consider the $\Delta$ contribution to the parity-violating potential up to the third order $\mathcal{O}(Q)$, we need only the lowest order chiral Lagrangians.

For the $\pi N N$ interaction, the PC part is

$$
\mathcal{L}_{\pi N, P C}^{(0)}=\bar{N}\left[i v \cdot \mathcal{D}+2 g_{A}^{0} S \cdot A\right] N
$$

where

$$
\begin{gathered}
\mathcal{D}_{\mu}=D_{\mu}+V_{\mu}, \quad V_{\mu}=\frac{1}{2}\left(\xi D_{\mu} \xi^{\dagger}+\xi^{\dagger} D_{\mu} \xi\right) \\
A_{\mu}=-\frac{i}{2}\left(\xi D_{\mu} \xi^{\dagger}-\xi^{\dagger} D_{\mu} \xi\right)=-\frac{D_{\mu} \pi}{F_{\pi}}+\mathcal{O}\left(\pi^{3}\right) \\
\xi=\exp \left(\frac{i \pi^{a} \tau^{a}}{2 F_{\pi}}\right)=\exp \left(\frac{i \pi}{F_{\pi}}\right) .
\end{gathered}
$$

Here $V_{\mu}$ and $A_{\mu}$ are the chiral connection and the axial field respectively. $v_{\mu}$ is the velocity and $S_{\mu}$ is the PauliLubanski spin vector. $F_{\pi}=92.4 \mathrm{MeV}$ is the pion decay constant and $\tau^{a}$ is the Pauli matrix. Here $g_{A} \simeq 1.27$ is the nucleon axial vector coupling constant.

The PV part is

$$
\begin{aligned}
\mathcal{L}_{\pi N, P V}^{(-1)} & =-\frac{h_{\pi}^{1} F_{\pi}}{2 \sqrt{2}} \bar{N} X_{-}^{3} N \\
& =-i h_{\pi}^{1}\left(\bar{p} n \pi^{+}-\bar{n} p \pi^{-}\right)+\ldots,
\end{aligned}
$$

where

$$
X_{-}^{3}=\xi^{+} \tau^{3} \xi-\xi \tau^{3} \xi^{+},
$$

and $h_{\pi}^{1} \sim 10^{-7}$ is the weak coupling constant. The ellipsis denotes terms involving more pions.

For the part containing $\Delta$, the leading Lagrangian reads 42 ]

$$
\begin{aligned}
\mathcal{L}_{\pi N \Delta, P C}^{(0)}= & -i \bar{T}^{\mu a} v \cdot D^{a b} T_{\mu}^{b}+\delta \bar{T}^{\mu a} T_{\mu}^{a} \\
& +2 g_{\pi N \Delta}\left(\bar{T}^{\mu a} A_{\mu}^{a} N+\bar{N} A_{\mu}^{a} T^{\mu a}\right),
\end{aligned}
$$

where $\delta=m_{\Delta}-m_{N}, A_{\mu}^{a}=\frac{1}{2} \operatorname{Tr}\left(A_{\mu} \tau^{a}\right)$ and $T^{\mu}$ represents $\Delta$ fields with

$$
\begin{gathered}
T_{\mu}^{1}=\frac{1}{\sqrt{2}}\left(\begin{array}{c}
\Delta^{++}-\Delta^{0} / \sqrt{3} \\
\Delta^{+} / \sqrt{3}-\Delta^{-}
\end{array}\right)_{\mu}, \\
T_{\mu}^{2}=\frac{i}{\sqrt{2}}\left(\begin{array}{c}
\Delta^{++}+\Delta^{0} / \sqrt{3} \\
\Delta^{+} / \sqrt{3}+\Delta^{-}
\end{array}\right)_{\mu}, \\
T_{\mu}^{3}=-\sqrt{\frac{2}{3}}\left(\begin{array}{c}
\Delta^{+} \\
\Delta^{0}
\end{array}\right)_{\mu} .
\end{gathered}
$$

In this Lagrangian, we have used $\mathcal{C}=\sqrt{2} g_{\pi N \Delta}$ with the language in Ref. 52, 53]. The quark model gives the relation $g_{\pi N \Delta}=\frac{3 \sqrt{2}}{5} g_{A}$. Since the $\mathrm{PV} \pi N \Delta$ part contributes to the $\mathrm{PV}$ potential beyond the order of $\mathcal{O}(Q)$ 22], we do not consider it here.

\section{III. $\triangle$ CONTRIBUTION TO PV NN POTENTIAL}

Because the $\mathrm{PV}$ contribution is tiny, one $\mathrm{PV}$ vertex is enough for the present study. The intermediate $\Delta$ contribution to parity-violating potential is presented in Fig. 1. We employ the counting scheme of SSE and truncate the expansion at the order $\mathcal{O}(Q)$. To this order, the triangle diagrams do not contribute. In the case without the $\Delta$ contribution, the box diagrams are two-particle reducible. Now the diagrams are all two-particle irreducible (2PI). That is, the diagrams in Fig. 1 will not induce double counting problem. In the following, we calculate the effective potentials in detail.

First, we consider the cross diagrams (a)-(d) in Fig. 1. From the vertices, one can construct four cases of transitions which include charge-conserving cases $p p \rightarrow$ 


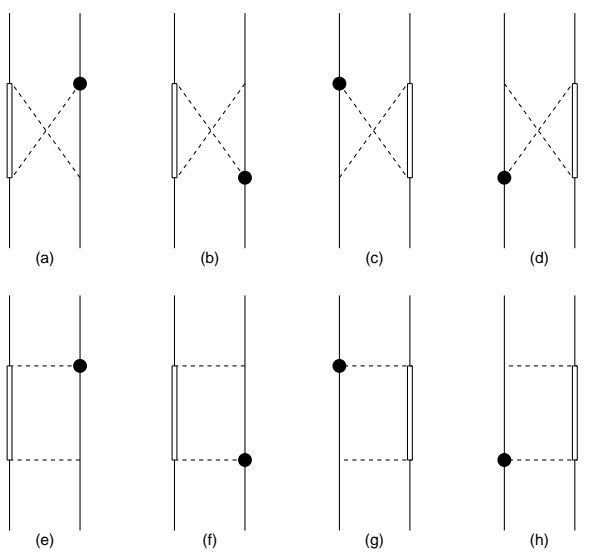

FIG. 1: Diagrams for intermediate $\Delta$ contribution to PV NN potential. The dotted lines are pions. The full lines represent nucleon while the double lines represent $\Delta$ states. Vertices with black dot mean the parity-violating $\pi N$ interaction.

$p p, n n \rightarrow n n$ and $p n \rightarrow p n(n p \rightarrow n p)$ and chargechanging case $p n \rightarrow n p(n p \rightarrow p n)$.

For $p p \rightarrow p p$, the sum of (a) and (b) gives

$$
i T=-i \frac{4 \sqrt{2}}{9} \frac{g_{\pi N \Delta}^{2} g_{A} h_{\pi}^{1}}{\Lambda_{\chi}^{2} F_{\pi}} Z(Q) \bar{p}\left[S_{1} \cdot q, S_{1 \mu}\right] p \bar{p} S_{2}^{\mu} p
$$

where $q=p_{1}-p_{1}^{\prime}=p_{2}^{\prime}-p_{2}, Q^{2}=-q^{2} \approx \mathbf{q}^{2}, \Lambda_{\chi}=4 \pi F_{\pi}$ and

$$
Z(Q)=2 L(Q)+\frac{\pi}{2 \delta}\left(4 m_{\pi}^{2}+Q^{2}\right) A(Q)-\frac{2}{\delta} B(Q),
$$

with

$$
\begin{aligned}
& L(Q)=\frac{\sqrt{4 m_{\pi}^{2}+Q^{2}}}{Q} \ln \frac{Q+\sqrt{4 m_{\pi}^{2}+Q^{2}}}{2 m_{\pi}} \\
& A(Q)=\frac{1}{2 Q} \arctan \frac{Q}{2 m_{\pi}} \\
& B(Q)=\int_{0}^{1} d y \int_{\delta}^{\infty} d \lambda \frac{m_{\pi}^{2}-\delta^{2}+y(1-y) Q^{2}}{\lambda^{2}+m_{\pi}^{2}-\delta^{2}+y(1-y) Q^{2}}(8)
\end{aligned}
$$

In calculating the loop integrals, we have used the dimensional regularization. The divergent part could be absorbed by the renormalization of the counter-terms at the same chiral order. Here we only retain the non-analytic terms.

For $n n \rightarrow n n$ channel, the sum of (a) and (b) is

$$
i T=i \frac{4 \sqrt{2}}{9} \frac{g_{\pi N \Delta}^{2} g_{A} h_{\pi}^{1}}{\Lambda_{\chi}^{2} F_{\pi}} Z(Q) \bar{n}\left[S_{1} \cdot q, S_{1 \mu}\right] n \bar{n} S_{2}^{\mu} n .
$$

This result is similar to the $p p \rightarrow p p$ channel. Similarly, one gets contributions from the mirror diagrams (c) and (d). Since the initial particles are identical, the operator form for these two channels will generate (a), (b) and the mirror diagrams (c), (d) simultaneously.
Compared with the case without the $\Delta$ contribution [31], there is an additional channel $p n \rightarrow p n$. The sum of (a)-(d) in the operator form reads

$$
\begin{aligned}
i T= & i \frac{2 \sqrt{2}}{3} \frac{g_{\pi N \Delta}^{2} g_{A} h_{\pi}^{1}}{\Lambda_{\chi}^{2} F_{\pi}} Z(Q)\left\{\bar{N}\left[S \cdot k, S_{\mu}\right] \tau_{3} N \bar{N} S^{\mu} N\right. \\
& \left.-\bar{N}\left[S \cdot k, S_{\mu}\right] N \bar{N} S^{\mu} \tau_{3} N\right\} .
\end{aligned}
$$

Here $k$ is the initial momentum minus the final momentum for a nucleon line.

After combining the above three channels, we get

$$
\begin{array}{r}
i \frac{4 \sqrt{2}}{9} \frac{g_{\pi N \Delta}^{2} g_{A} h_{\pi}^{1}}{\Lambda_{\chi}^{2} F_{\pi}} Z(Q) \bar{N}\left[S \cdot k, S_{\mu}\right] \tau_{3} N \bar{N} S^{\mu} N \\
-i \frac{8 \sqrt{2}}{9} \frac{g_{\pi N \Delta}^{2} g_{A} h_{\pi}^{1}}{\Lambda_{\chi}^{2} F_{\pi}} Z(Q) \bar{N}\left[S \cdot k, S_{\mu}\right] N \bar{N} S^{\mu} \tau_{3} N .
\end{array}
$$

For the charge-changing case $p n \rightarrow n p$, the sum of diagrams (a)-(d) gives

$$
-\frac{\sqrt{2}}{6} \frac{g_{\pi N \Delta}^{2} g_{A} h_{\pi}^{1}}{\Lambda_{\chi}^{2} F_{\pi}} Y(Q) \epsilon^{i j 3} \bar{N} \tau^{i} N \bar{N} \tau^{j} \boldsymbol{\sigma} \cdot \mathbf{k} N,
$$

where

$$
Y(Q)=2 L(Q)+\frac{2 \pi}{3 \delta}\left(2 m_{\pi}^{2}+Q^{2}\right) A(Q)-\frac{2}{\delta} C(Q)
$$

with

$$
\left.C(Q)=\int_{0}^{1} d y \int_{\delta}^{\infty} d \lambda \frac{m_{\pi}^{2}-\delta^{2}+\frac{4}{3} y(1-y) Q^{2}}{\lambda^{2}+m_{\pi}^{2}-\delta^{2}+y(1-y) Q^{2}} 14\right)
$$

Next, we consider the box diagrams (e)-(h) in Fig. 1 There are also four cases: charge-conserving processes $n p \rightarrow n p(p n \rightarrow p n), p p \rightarrow p p$ and $n n \rightarrow n n$ and chargechanging process $n p \rightarrow p n(p n \rightarrow n p)$.

For the channel $n p \rightarrow n p$, the sum of the diagrams (e)-(h) gives

$$
\begin{aligned}
i T= & i \frac{2 \sqrt{2}}{9} \frac{g_{\pi N \Delta}^{2} g_{A} h_{\pi}^{1}}{\Lambda_{\chi}^{2} F_{\pi}} W(Q)\left\{\bar{N}\left[S \cdot k, S_{\mu}\right] \tau_{3} N \bar{N} S^{\mu} N\right. \\
& \left.-\bar{N}\left[S \cdot k, S_{\mu}\right] N \bar{N} S^{\mu} \tau_{3} N\right\}
\end{aligned}
$$

where

$$
W(Q)=2 L(Q)-\frac{\pi}{2 \delta}\left(4 m_{\pi}^{2}+Q^{2}\right) A(Q)-\frac{2}{\delta} B(Q) .
$$

In calculating the amplitudes, we use the following formula

$$
\frac{1}{v \cdot k+i \epsilon}=-\frac{1}{-v \cdot k+i \epsilon}-2 \pi i \delta(v \cdot k) .
$$

In the case without the $\Delta$ contribution, the part from the $\delta$ function was subtracted to separate the contributions from the iterated one-pion exchange and those from the 
irreducible two-pion exchange. Now this part is included because the diagram is $2 \mathrm{PI}$.

The diagrams (e) and (f) result in

$$
i T=-i \frac{4 \sqrt{2}}{3} \frac{g_{\pi N \Delta}^{2} g_{A} h_{\pi}^{1}}{\Lambda_{\chi}^{2} F_{\pi}} W(Q) \bar{p}\left[S_{1} \cdot q, S_{1 \mu}\right] p \bar{p} S_{2}^{\mu} \not(18)
$$

for the channel $p p \rightarrow p p$ and

$$
i T=i \frac{4 \sqrt{2}}{3} \frac{g_{\pi N \Delta}^{2} g_{A} h_{\pi}^{1}}{\Lambda_{\chi}^{2} F_{\pi}} W(Q) \bar{n}\left[S_{1} \cdot q, S_{1 \mu}\right] n \bar{n} S_{2}^{\mu} \eta
$$

for $n n \rightarrow n n$.

After combining these results, we get the chargeconserving amplitude from box diagrams

$$
\begin{aligned}
& -i \frac{4 \sqrt{2}}{9} \frac{g_{\pi N \Delta}^{2} g_{A} h_{\pi}^{1}}{\Lambda_{\chi}^{2} F_{\pi}} W(Q) \bar{N}\left[S \cdot k, S_{\mu}\right] \tau_{3} N \bar{N} S^{\mu} N \\
& -i \frac{8 \sqrt{2}}{9} \frac{g_{\pi N \Delta}^{2} g_{A} h_{\pi}^{1}}{\Lambda_{\chi}^{2} F_{\pi}} W(Q) \bar{N}\left[S \cdot k, S_{\mu}\right] N \bar{N} S^{\mu} \tau_{3} N .
\end{aligned}
$$

For the charge-changing case $n p \rightarrow p n$, one sums the amplitudes from (e)-(h) and gets

$$
-\frac{\sqrt{2}}{6} \frac{g_{\pi N \Delta}^{2} g_{A} h_{\pi}^{1}}{\Lambda_{\chi}^{2} F_{\pi}} X(Q) \epsilon^{i j 3} \bar{N} \tau^{i} N \bar{N} \tau^{j} \boldsymbol{\sigma} \cdot \mathbf{k} N
$$

where

$$
X(Q)=2 L(Q)-\frac{2 \pi}{3 \delta}\left(2 m_{\pi}^{2}+Q^{2}\right) A(Q)-\frac{2}{\delta} C(Q) .
$$

After combing Eqs. (11), (12), (20) and (21), we finally get the $\Delta$ contribution to nuclear parity violation

$$
\begin{aligned}
& -\frac{\sqrt{2}}{9} \frac{g_{\pi N \Delta}^{2} g_{A} h_{\pi}^{1}}{\Lambda_{\chi}^{2} F_{\pi}}[W(Q)-Z(Q)] \epsilon^{i j k} N^{\dagger} k^{i} \sigma^{j} \tau_{3} N N^{\dagger} \sigma^{k} N \\
& -\frac{2 \sqrt{2}}{9} \frac{g_{\pi N \Delta}^{2} g_{A} h_{\pi}^{1}}{\Lambda_{\chi}^{2} F_{\pi}}[W(Q)+Z(Q)] \epsilon^{i j k} N^{\dagger} k^{i} \sigma^{j} N N^{\dagger} \sigma^{k} \tau_{3} N \\
& -\frac{\sqrt{2}}{6} \frac{g_{\pi N \Delta}^{2} g_{A} h_{\pi}^{1}}{\Lambda_{\chi}^{2} F_{\pi}}[X(Q)+Y(Q)] \epsilon^{i j 3} N^{\dagger} \tau^{i} N N^{\dagger} \tau^{j} \boldsymbol{\sigma} \cdot \mathbf{k} N(23)
\end{aligned}
$$

Acordingly, one gets the PV potential

$$
\begin{aligned}
V= & -\frac{i}{\Lambda_{\chi}^{3}}\left\{\tilde{C}_{2}^{\Delta}(Q) \frac{\tau_{1}^{z}+\tau_{2}^{z}}{2}\left(\boldsymbol{\sigma}_{\mathbf{1}} \times \boldsymbol{\sigma}_{\mathbf{2}}\right) \cdot \mathbf{q}\right. \\
& \left.+C_{6}^{\Delta}(Q)\left(\boldsymbol{\tau}_{\mathbf{1}} \times \boldsymbol{\tau}_{\mathbf{2}}\right)^{z}\left(\boldsymbol{\sigma}_{\mathbf{1}}+\boldsymbol{\sigma}_{\mathbf{2}}\right) \cdot \mathbf{q}\right\},
\end{aligned}
$$

where

$$
\begin{aligned}
\tilde{C}_{2}^{\Delta}(Q)= & \frac{8 \sqrt{2}}{9} \pi g_{\pi N \Delta}^{2} g_{A} h_{\pi}^{1}\left[4 L(Q)-\frac{4}{\delta} C(Q)\right], \\
C_{6}^{\Delta}(Q)= & -\frac{2 \sqrt{2}}{3} \pi g_{\pi N \Delta}^{2} g_{A} h_{\pi}^{1}[8 L(Q) \\
& \left.-\frac{\pi}{\delta}\left(4 m_{\pi}^{2}+Q^{2}\right) A(Q)-\frac{8}{\delta} B(Q)\right] .
\end{aligned}
$$

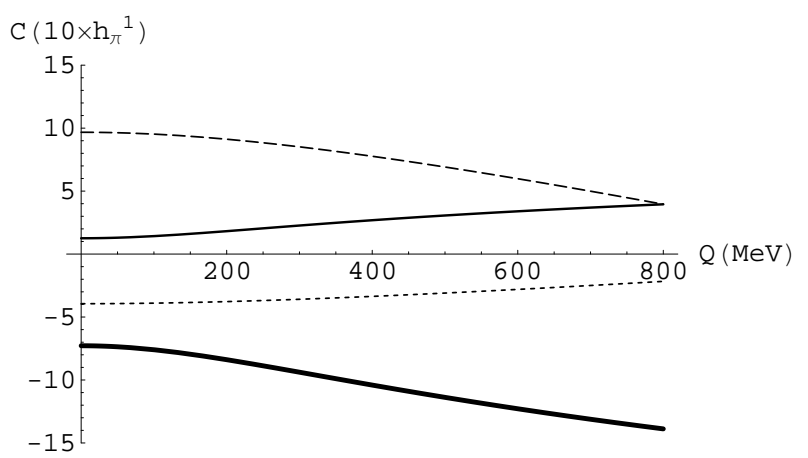

FIG. 2: The momentum dependence of coefficients in the PV two-pion exchange potentials: $\tilde{C}_{2}^{2 \pi}(Q)$ (thick solid line), $C_{6}^{2 \pi}(Q)$ (thin solid line), $\tilde{C}_{2}^{\Delta}(Q)$ (dash line) and $C_{6}^{\Delta}(Q)$ (dotted line).

\section{DISCUSSIONS}

In short summary, we have calculated the $\Delta$ contribution to the parity-violating nucleon-nucleon potential. The $\Delta$ resonance couples to the nucleon and pion strongly. A nucleon may be excited and transformed into a virtual $\Delta$ quite easily. The $\Delta$ will certainly contribute to the hadronic parity violation in nuclear processes. In this work, we employ the small scale expansion formalism and extend the former investigation of PV NN interaction in EFT in Ref. 31] through the inclusion of the $\Delta$ contribution. To the next-next-leading order, the new potential contains no more unknown PV coupling constants. The only new parameter is the strong coupling constant $g_{\pi N \Delta}$, which is known from the decay width of the $\Delta$ baryon.

The structure of the obtained potential is similar to the medium-range potential derived in Ref. [6, 31]. For comparison, we plot the momentum dependence of coefficients $\tilde{C}_{2}^{\Delta}(Q), C_{6}^{\Delta}(Q), \tilde{C}_{2}^{2 \pi}(Q)=-8 \sqrt{2} \pi g_{A}^{3} h_{\pi}^{1} L(Q)$ and $C_{6}^{2 \pi}(Q)=-\sqrt{2} \pi g_{A} h_{\pi}^{1} L(Q)+\sqrt{2} \pi[3 L(Q)-H(Q)] g_{A}^{3} h_{\pi}^{1}$ with $H(Q)=\frac{4 m_{\pi}^{2}}{4 m_{\pi}^{2}+Q^{2}} L(Q)$ in Fig. 2. We take $m_{\pi}=135$ $\mathrm{MeV}, \delta=294 \mathrm{MeV}, g_{A}=1.27, g_{\pi N \Delta}=\frac{3 \sqrt{2}}{5} g_{A}$. From the figure, one notes $C_{6}^{\Delta}(Q)$ is bigger than $C_{6}^{2 \pi}(Q)$ at small momentum. It is also important to note that $\tilde{C}_{2}^{\Delta}(Q)$ and $\tilde{C}_{2}^{2 \pi}(Q)$ are comparable in magnitude but they have opposite signs! Therefore it is highly desirable to include the new parity violating nucleon-nucleon arising from the $\Delta$ correction in the future theoretical calculation of PV observables in the hadronic weak interaction processes. 


\section{Acknowledgments}

We thank Prof. B. Desplanques for helpful discussions. This project was supported by the National Natu- ral Science Foundation of China under Grants 10625521 , 10721063 and 10775146, the China Postdoctoral Science Foundation (20070420526) and Ministry of Education of China.
[1] Tanner N, Phys. Rev., 1957, 107: 1203

[2] Wu C S et. al., Phys. Rev., 1957, 105: 1413

[3] Adelberger E G, Haxton W C, Annu. Rev. Nucl. Part. Sci., 1985, 35: 501

[4] Haxton W C, Wieman C E, Annu. Rev. Nucl. Part. Sci., 2001, 51: 261

[5] Holstein B R, Nucl.Phys. A, 2004, 737: 85

[6] Ramsey-Musolf M J, Page S A, Annu. Rev. Nucl. Part. Sci., 2006, 56: 1

[7] Berdoz A R et. al., Phys. Rev. Lett., 2001, 87: 272301

[8] Berdoz A R et. al., Phys. Rev. C, 2003, 68: 034004

[9] Gericke M T et. al., nucl-ex/0507005

[10] Stiliaris E, Eur. Phys. J. A, 2005, 24S2: 175

[11] Bass C D et. al., J. Res. Natl. Inst. Stand. Technol., 2005, 110: 205

[12] Micherdzinska A Z et. al., AIP Conf. Proc., 2006, 842: 799

[13] Michel F C, Phys. Rev., 1964, 133: B329

[14] Fischbach E, Phys. Rev., 1968, 170: 1398

[15] Tadic D, Phys. Rev., 1968, 174: 1694

[16] McKellar B H J, Pick P, Phys. Rev. D, 1973, 7260

[17] Desplanques B, Donoghue J F, Holstein B R, Ann. Phys. (N.Y.), 1980, 124: 449

[18] Holstein B R, Phys. Rev. D, 1981, 23: 1618

[19] Wood C S et. al., Science, 1997, 275: 1759

[20] Haxton W C, Liu C P, Ramsey-Musolf M J, Phys. Rev. C, 2002, 65: 045502

[21] Kaplan D B, Savage M J, Nucl. Phys. A, 1993, 556: 653

[22] Zhu S L, Puglia S J, Holstein B R, Ramsey-Musolf M J, Phys. Rev. D, 2001, 63: 033006

[23] Zhu S L, Puglia S J, Holstein B R, Ramsey-Musolf M J, Phys. Rev. C, 2001 64: 035502

[24] Zhu S L, Maekawa C M, Holstein B R, Ramsey-Musolf M J, Phys. Rev. Lett., 2001, 87: 201802

[25] Zhu S L, Maekawa C M, Sacco G, Holstein B R, RamseyMusolf M J, Phys. Rev. D, 2001, 65: 033001

[26] Savage M J, Springer R P, Nucl. Phys. A, 1998, 644: 235

[27] Savage M J, Springer R P, Nucl. Phys. A, 1999, 657: 457

[28] Savage M J, Springer R P, Nucl. Phys. A, 2001, 686: 413

[29] Kaplan D B, Savage M J, Springer R P, Wise M B, Phys. Lett. B, 1999, 449: 1
[30] Hyun C H, Park T S, Min D P, Phys. Lett. B, 2001, 516: 321

[31] Zhu Shi-Lin, Maekawa C M, Holstein B R, RamseyMusolf M J, van Kolck U, Nucl. Phys. A, 2005, 748: 435

[32] Weinberg S, Phys. Lett. B, 1990, 251: 288

[33] S. Weinberg, Nucl. Phys. B, 1991, 363: 3

[34] Liu C P, Phys. Rev. C, 2007, 75: 065501

[35] Hyun C H, Ando S, Desplanques B, Eur. Phys. A, 2007, 32: 513

[36] Epstein G N, Phys. Lett. B, 1977, 71: 267

[37] Silbar R R, Kloet W M, Kisslinger L, Dubach J, Phys. Rev. C, 1989, 40: 2218

[38] Kloet W M, Silbar R R, Tjon G A, Phys. Rev. C, 1989, 41: 2263

[39] Feldman G B, Crawford G A, Dubach J, Holstein B R, Phys. Rev. C, 1991, 43: 863

[40] Kaiser N, Phys. Rev. C, 2007, 76: 047001

[41] Niskanen J A, Partanen T M, Iqbal M J, arXiv: 0712.2399

[42] Hemmert T R, Holstein B R, Kambor J, J. Phys. G, 1998, 24: 1831

[43] Bernard V, Fearing H W, Hemmert T R, Meissner U G, Nucl. Phys. A, 1998, 635: 121

[44] Bernard V, Fearing H W, Hemmert T R, Meissner U G, Nucl. Phys. A, 1998, 642: 563

[45] Gellas G C, Hemmert T R, Ktorides C N, Poulis G I, Phys. Rev. D, 1999, 60: 054022

[46] Fettes N, Meissner U G, Nucl. Phys. A, 2001, 679: 629

[47] Puglia S J, Ramsey-Musolf M J, Zhu S L, Phys. Rev. D, 2001, 63: 034014

[48] Zhu S L, Puglia S, Ramsey-Musolf M J, Phys. Rev. D, 2001, 63: 034002

[49] Zhu S L, Sacco G, Ramsey-Musolf M J, Phys. Rev. D, 2002, 66: 034021

[50] Villadoro G, Phys. Rev. D, 2006, 74: 014018

[51] Liu Y R, Zhu S L, Eur. Phys. C, 2007 52: 177

[52] Jenkins E, Manohar A V, Phys. Lett. B, 1991, 255: 558

[53] Jenkins E, Manohar A V, Phys. Lett. B, 1991, 259: 353 